\title{
a-Synuclein pathology in Parkinson disease activates homeostatic NRF2 anti-oxidant response
}

\author{
Alberto Delaidelli, ${ }^{3,4}$, Mette Richner ${ }^{1 \dagger}$, Lixiang Jiang ${ }^{1}$, Amelia van der Laan ${ }^{1}$, Ida Bergholdt Jul Christiansen ${ }^{1}$, \\ Nelson Ferreira' ${ }^{1}$, Jens R. Nyengaard ${ }^{2}$, Christian B. Vægter ${ }^{1}$, Poul H. Jensen ${ }^{1}$, lan R. Mackenzie ${ }^{3}$, \\ Poul H. Sorensen ${ }^{3,4}$ and Asad Jan ${ }^{1 *}$ (D)
}

\begin{abstract}
Circumstantial evidence points to a pathological role of alpha-synuclein (aSyn; gene symbol SNCA), conferred by aSyn misfolding and aggregation, in Parkinson disease (PD) and related synucleinopathies. Several findings in experimental models implicate perturbations in the tissue homeostatic mechanisms triggered by pathological aSyn accumulation, including impaired redox homeostasis, as significant contributors in the pathogenesis of PD. The nuclear factor erythroid 2-related factor (NRF2/Nrf2) is recognized as 'the master regulator of cellular anti-oxidant response', both under physiological as well as in pathological conditions. Using immunohistochemical analyses, we show a robust nuclear NRF2 accumulation in post-mortem PD midbrain, detected by NRF2 phosphorylation on the serine residue 40 (nuclear active p-NRF2, S40). Curated gene expression analyses of four independent publicly available microarray datasets revealed considerable alterations in NRF2-responsive genes in the disease affected regions in PD, including substantia nigra, dorsal motor nucleus of vagus, locus coeruleus and globus pallidus. To further examine the putative role of pathological aSyn accumulation on nuclear NRF2 response, we employed a transgenic mouse model of synucleionopathy (M83 line, expressing the mutant human A53T aSyn), which manifests widespread aSyn pathology (phosphorylated aSyn; S129) in the nervous system following intramuscular inoculation of exogenous fibrillar aSyn. We observed strong immunodetection of nuclear NRF2 in neuronal populations harboring p-aSyn (S129), and found an aberrant anti-oxidant and inflammatory gene response in the affected neuraxis. Taken together, our data support the notion that pathological aSyn accumulation impairs the redox homeostasis in nervous system, and boosting neuronal anti-oxidant response is potentially a promising approach to mitigate neurodegeneration in PD and related diseases.
\end{abstract}

Keywords: Parkinson disease, Alpha-synuclein, Oxidative stress, NRF2

\footnotetext{
*Correspondence: ajan@aias.au.dk

${ }^{\dagger}$ Alberto Delaidelli and Mette Richner contributed equally

${ }^{1}$ Danish Research Institute of Translational Neuroscience (DANDRITE)Nordic-EMBL Partnership for Molecular Medicine, Department of Biomedicine, Aarhus University, Ole Worms Allé 3, 8000 Aarhus C, Denmark

Full list of author information is available at the end of the article
}

\begin{abstract}
Introduction
Parkinson disease (PD) is a major neurodegenerative cause of chronic dysfunction in the subcortical somatomotor system, which is frequently compounded by nonmotor symptoms of extranigral origin [1-3]. The disease is clinically characterized by the cardinal features of resting tremor, bradykinesia, rigidity and gait apraxia $[1,2]$. The neuropathology of PD is principally defined by the loss of dopaminergic neurons in the midbrain substantia
\end{abstract} original author(s) and the source, provide a link to the Creative Commons licence, and indicate if changes were made. The images or other third party material in this article are included in the article's Creative Commons licence, unless indicated otherwise in a credit line to the material. If material is not included in the article's Creative Commons licence and your intended use is not permitted by statutory regulation or exceeds the permitted use, you will need to obtain permission directly from the copyright holder. To view a copy of this licence, visit http://creativecommons.org/licenses/by/4.0/. The Creative Commons Public Domain Dedication waiver (http://creativeco mmons.org/publicdomain/zero/1.0/) applies to the data made available in this article, unless otherwise stated in a credit line to the data. 
nigra (SN)-pars compacta, and immunoreactive deposits of $\alpha$-synuclein (aSyn; gene symbol SNCA) protein across multiple regions in the central nervous system (CNS) $[1,2,4]$. Pathological aSyn deposition in the CNS, termed Lewy body (LB) pathology, is also seen in other neurodegenerative syncleinopathies including Dementia with Lewy bodies (DLB) and Multiple system atrophy (MSA) [5]. In the vast majority of PD cases, the disease is of idiopathic (non-inheritable) origin, and genetic factors underlie $5-10 \%$ of clinically diagnosed PD [2]. Rare missense mutations in SNCA that result in N-terminal amino acid substitutions in aSyn, or multiplications in $S N C A$ gene locus are recognized etiological factors in the autosomal-dominant forms of PD [2, 6, 7]. Additionally, mutations in several other genes (of autosomal-dominant or recessive inheritance) have been discovered to cause rare forms of familial PD, underscoring the complex etiology of the disease $[1,2]$. Furthermore, the occurrence of distinct neurodegenerative lesions and progressive aSyn pathology in PD point to the selective vulnerability of specific subcortical nuclei, with relative sparing of other brain regions $[4,8,9]$.

Experimental models based on the genetic aberrations in familial PD have revealed a number of candidate mechanisms that are potentially relevant to neurodegeneration, and for the development of mechanism-based therapies in PD. These studies implicate perturbed cellular homeostasis caused by several biochemical alterations including defective autophagic flux, endoplasmic reticulum (ER) stress, calcium dyshomeostasis and mitochondrial impairment [10-13]. Although predominantly studied as a sequelae to aSyn aggregation $[11,13]$, the relevance of these mechanisms is also supported by findings in experimental paradigms based on other genetic factors causing PD. For instance, mutations in leucine-rich repeat kinase 2 (LRRK2) - the most common cause of the autosomal dominant PD-are associated functional alterations such as impaired vesicular trafficking and cytoskeleton dynamics, defective autophagy and lysosomal degradation, and mitochondrial dysfunction $[14,15]$. Furthermore, mutations of Parkin (PARK2; an E3 ubiquitin ligase), the protein deglycase DJ-1 (PARK7) and the PTEN-induced putative kinase 1 (PINK1) cause functional deficits affecting the autophagic flux of damaged mitochondria and neuroprotective response against oxidative stress [12]. Moreover, inadequate mitochondrial complex I activity, as a result of aSyn aggregation or in chemically induced experimental parkinsonism, has been implicated in the impaired energy production, and in the oxidative damage by increased free radical production $[16,17]$. Similarly, post-mortem studies in PD implicate aberrant ROS homeostasis, lipid peroxidation, protein nitration and nucleic acid oxidation as significant disease associations $[18,19]$. Lastly, cellular oxidative stress has been shown to exacerbate aSyn misfolding and aggregation, thus triggering a vicious cycle that culminates in cytotoxicity and further redox dyshomeostasis [20]. Although, the nature of the final common pathway(s) that triggers neurodegeneration in PD remains debatable, an interplay of the above mentioned genetic factors and environmental triggers are thought to blunt the protective tissue homeostatic response against the deleterious effects of proteotoxic and oxidative stress [12, 13].

In this context, the nuclear factor erythroid 2-related factor 2 (protein, NRF2/Nrf2; gene symbol NFE2L2), a member of basic leucine zipper (bZIP) protein family, plays a crucial role in the cellular adaptive response under oxidative and metabolic stress $[21,22]$. Under resting metabolic conditions, NRF2 is sequestered in the cytoplasm by its inhibitor, Kelch-Like ECH-Associated Protein-1 (protein, Keap1; gene symbol KEAP1), which also mediates the proteosomal degradation of NRF2 [22]. A mismatch between the production of reactive oxygen species (ROS) free radicals and ROS scavenging mechanisms induces the phosphorylation of NRF2 at serine 40 residue (S40) and disrupts the NRF2-Keap1 complex. As a result, there is increased cytoplasm-to-nucleus translocation of NRF2, and subsequent transactivation of the pathways involved in antioxidant, anti-inflammatory and xenobiotic defence (including NRF2 auto-regulation) [22]. In addition to the canonical regulation via Keap1 mediated autophagic degradation, NRF2 activity is also influenced by several factors including post-translational modifications, epigenetic factors, microRNAs and metabolic adaptations under nutrient stress [22-24]. Burgeoning evidence points to the relevance of aberrant NRF2 activity in the pathogenesis of neurodegenerative diseases, including PD $[25,26]$. First, there is a correlative decline in NRF2 activity with age, which is the strongest risk factor in the common neurodegenerative diseases [27]. Second, neuropathological studies suggest increased nuclear localization of total NRF2 protein in the postmortem SN of PD patients, in contrast to the predominant cytosolic localization in controls [26]. Third, single nucleotide polymorphisms (SNPs) in NFE2L2 or the promoter region are associated with altered disease risk or the age of PD onset [28]. Therefore, understanding the role of NRF2 response in the pathophysiology of neurodegeneration in PD and related diseases, and investigating the therapeutic impact of boosting NRF2 anti-oxidant response has gained significant interest $[16,25,28]$.

In this report, we show increased nuclear localization of NRF2 in post-mortem PD midbrain in the presence of Lewy related aSyn pathology, as detected by immunonhistochemical (IHC) detection of phosphorylated NRF2 on the residue serine-40 (p-NRF2, S40), a 
robust post-translational modification associated with the nuclear accumulation of NRF2 [29-31]. By analyzing four independent microarray studies in PD [32-35], we also report altered expression of NRF2-responsive genes in PD affected brain regions. In order to establish the role of pathological aSyn accumulation to altered NRF2 response, we performed IHC and gene expression analyses in a transgenic mouse model of synucleinopathy expressing the PD-associated mutant Ala53Thr (A53T) aSyn (the M83 line) [36, 37]. In this rodent model, widespread aSyn pathology (phosphorylated aSyn, serine-129; $\mathrm{p}$-aSyn, S129) in the CNS is ectopically induced by the intramuscular inoculation of pre-formed fibrillar (PFF) aSyn in the hindlimb. Specifically, the intracerebral aSyn pathology is predominantly observed in the brainstem regions around 50-70 days post-injection, with relative paucity in the forebrain areas [36, 37]. Our data show considerable enrichment of nuclear NRF2 (p-NRF2, S40) in the brain regions harboring aSyn pathology (p-aSyn, S129), and distinct alterations in the NRF2-dependent anti-oxidant and inflammatory gene expression response in this prion-like model of synucleionoapthy. Taken together, our data provide fruitful insights into the putative role of NRF2 stress adaptive signaling in PD in relation to pathological aSyn accumulation, and strengthen the rationale for boosting NRF2-anti-oxidant response towards mitigating the deleterious effects of proteopathic stress.

\section{Methods}

\section{Human studies}

Human tissue processing and Immunohistochemistry (IHC)

Five-micrometer formalin-fixed paraffin embedded post-mortem sections from midbrains of control or PD patients were provided by the laboratory of IM (coauthor), as approved by the University of British Columbia Ethics Committee. Anonymized brain sections from 3 control individuals and 5 clinically and pathologically confirmed PD patients were used in these experiments (Additional file 1: Table S1).

IHC on brain sections from human tissue was performed after deparaffinization and antigen retrieval. The following antibodies were employed to stain serial tissue sections, as indicated: antibody against phospho-alpha synuclein (p-aSyn, S129; 81A monoclonal; EMD Millipore, \#MABN826; dilution 1:1000) [38], and antibody against phospho-NRF2 (p-NRF2, S40; EP1809Y monoclonal; abcam \# ab76026); dilution 1:400) using the alkaline phosphatise conjugated streptavidin-biotin $\mathrm{ABC}$ kit (Vector Labs, \#AK-5000). For destaining/bleaching neuromelanin in substantia nigra in the midbrain sections, the IHC protocol was modified slightly, as described [38]. Briefly, sections mounted on slides were incubated in a
$60{ }^{\circ} \mathrm{C}$ degrees oven for $30 \mathrm{~min}$ and then were transferred into ambient distilled water. Then, the slides were placed in $0.25 \%$ potassium permanganate solution for $5 \mathrm{~min}$. Subsequently, the slides were rinsed with distilled water. This was followed by incubation in 5\% oxalic acid until sections became clear. A final rinse in distilled water was performed before proceeding with the normal IHC staining as described above. Sections were counterstained with hematoxylin (Vector Labs, \#H-3401). High resolution panoramic images of tissue sections for IHC analyses were acquired using a Leica Aperio digital slide scanner. IHC staining for p-NRF2 (S40) and p-aSyn (S129) was quantified by manual counting of the DAB (3,3'-diaminobenzidine; Vector Labs, \#SK-4100) positive cells.

Microarray analyses. Normalized gene expression data from the following trancriptomics datasets was accessed on the Gene Expression Omnibus (GEO) [39] repository of the National Center for Biotechnology Information (NCBI): 1) GSE7621 (substantia nigra-SN; Controls, $\mathrm{n}=9 ; \mathrm{PD}, \mathrm{n}=16$ ) [34], 2) GSE43490 (substantia nigra-SN, dorsal motor nucleus of vagus-DMX and locus coeruleus$L C$; Controls, $\mathrm{n}=5-7$; PD, $\mathrm{n}=8$ ) [32], 3) GSE20146 (globus pallidus, interna-GPi; Controls, $\mathrm{n}=10 ; \mathrm{PD}, \mathrm{n}=10$ ) [35] and 4) GSE26927 (substantia nigra-SN; Controls, $\mathrm{n}=7$; PD, $\mathrm{n}=12$ ) [33]. Unique probe identities for the transcripts, and additional microarray platform information is provided in Additional file 1: Table S2.

\section{Animal studies}

\section{Animal husbandry}

Transgenic M83 mice [B6;C3-Tg(PrnpSNCA*A53T)83Vle/J] [36] were housed at the Bartholin animal facility at Aarhus University in accordance with Danish regulations and the European Communities Council Directive for laboratory animals, under the authorization \#2017-15-0201-01203 issued to PHJ (coauthor). The mice we housed under $12 \mathrm{~h} \mathrm{light/dark} \mathrm{cycle}$ and fed with regular chow diet ad libitum. The experiments were performed using both male and female mice.

\section{Intramuscular injection of preformed aSyn fibrils}

Mouse aSyn fibrils were prepared and characterized in vitro (for purity, biophysical properties and biological activity) essentially as described [40]. Homozy-

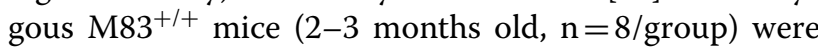
bilaterally inoculated with a single injection $(5 \mu \mathrm{l})$ of recombinant mouse aSyn preformed fibrils (PFF, $2 \mathrm{mg} /$ $\mathrm{mL}$ in phosphate buffered saline-PBS) - or PBS vehicle-into the hindlimb biceps femoris (using a $10-\mu \mathrm{L}$ Hamilton syringe with a 25 -gauge needle) under isoflurane $(1-5 \%)$ anesthesia $[38,40]$. Separate syringes were used for each type of inoculum (PBS or PFF) to avoid 
cross-contamination. After the injection, the mice were allowed to recover and returned to their original housing cages.

\section{Tissue collection}

Mice were euthanized with an overdose of sodium pentobarbital (150 mg/kg, intraperitoneal) and perfused with ice-cold PBS pH 7.4 containing phosphatase inhibitors (25 mM $\beta$-glycerolphosphate, $5 \mathrm{mM} \mathrm{NaF}, 1 \mathrm{mM} \mathrm{Na} 3 \mathrm{VO} 4$, $10 \mathrm{mM}$ Na-pyrophosphate). Brains were collected and one hemisphere was processed for IHC (see below). The contralateral hemisphere was further microdissected under the microscope to isolate the brain region of interest on ice-cold sterile filtered PBS supplemented with $10 \mathrm{mM}$ D-glucose (ThermoFisher \# A2494001), snap frozen in liquid nitrogen, and stored at $-80{ }^{\circ} \mathrm{C}$ for total RNA extraction (see below).

\section{IHC}

IHC on $10 \mu \mathrm{m}$ thick sections from formalin fixed paraffin embedded tissue was performed after deparaffinization and antigen retrieval in citrate buffer $\mathrm{pH}$ 6.0. Following primary antibodies were employed: phospho- $\alpha$ Syn-S129 (11A5 monoclonal, kind gift to PHJ by Imago Pharmaceuticals-1:1000) [40] and phospho-NRF2-S40 (rabbit polyclonal, ThermoFisher \#PA5-67520-1:500). For IHC, DAB (Electron Microscopy Sciences \#13082) chromogen detection was performed following incubation with biotin conjugated secondary antibodies and Extra-Avidin peroxidise (Sigma \#E2886-1:200). Sections were counterstained with hematoxylin (Vector Labs, \#H-3401). High resolution views were obtained using Olympus VS120 digital slide scanner and 5-40 $\times$ views were extracted using OlyVia software (Olympus). Panoramic digital slide scans were mapped onto Mouse Brain Atlas to neuroanatomically define the regions/nuclei (Paxinos and Franklin's The Mouse Brain in Stereotaxic Coordinates, Elsevier Publishing, $4^{\text {th }}$ Ed.) [41].

\section{Quantitative RT-PCR}

Total RNA was extracted from the fresh frozen tissue using QIAzol lysis reagent (Qiagen, \#74134) and purified using a commercial kit (Qiagen, \#74134). cDNA was synthesized from $500 \mathrm{ng}$ of total RNA using high capacity reverse transcriptase kit (Applied Biosystems, \#4368814). RT-qPCR was performed using SYBR green (Thermo Fisher, \#4385616) under standard conditions with unique primer pairs (Additional file 1: Table S3) in duplicate samples. The data were analyzed by relative $\Delta \Delta \mathrm{CT}$ quantification method using the murine glyceraldehyde 3-phosphate dehydrogenase (Gapdh) cycle (CT) values as the internal reference in each sample [42].

\section{Cell culture \\ aSyn plasmid transient overexpression and Nrf2 anti-oxidant response in cultured $\mathrm{N} 2 \mathrm{~A}$ cultures}

Mouse neuroblastoma (N2A) cells were obtained from ATCC (\#CCL-131), and maintained in DMEM (4.5 g/L glucose; Gibco, \#11965-084) supplemented with 1\% antibiotic-antimycotic solution (Gibco, \#15240062) and $10 \%$ Fetal Bovine Serum (FBS). The cells were cultured in 6-well (500, 000 cells/well) 12-well (250000 cells/ well) or 96 -well (50000 cells/well) plates. DNA plasmid transfections were performed using Lipofectamine 2000 (Invitrogen, \#11668019) according to the recommended procedures. After $24 \mathrm{~h}$, cells were briefly washed with phosphate-buffer saline (PBS) and allowed to differentiate into neurons in a modified culture medium containing DMEM (Gibco, \#21969035) supplemented with $500 \mu \mathrm{M}$ L-glutamine, $1 \%$ antibiotic-antimycotic, 2\% FBS and $500 \mu \mathrm{M}$ Dibutyryladenosine $3^{\prime}, 5^{\prime}$-cyclic monophosphate (db cAMP; Sigma, \#D0627) [38]. Unless indicated otherwise, differentiated N2A cells which were mock transfected, or transfected with AS plasmids (ASyn-WT or ASyn-A53T; Addgene plasmid \#40824 and \#40825 respectively) were used in the assays after $72-76 \mathrm{~h}$ post-transfection. Cellular ROS measurements were performed after incubation with $5 \mu \mathrm{g} / \mathrm{mL}$ 2,7-dichlorofluorescein diacetate-H2DCFDA (ThermoFisher \#D399) for $30 \mathrm{~min}$ in fresh medium ( \pm pre-treatment with hydrogen peroxide, $\mathrm{H}_{2} \mathrm{O}_{2} ; 250 \mu \mathrm{M}$ for $2 \mathrm{~h}$ ) according to the manufacturer's protocol, and essentially as described $[38,43]$. Anti-oxidant response element (ARE, Nrf2) promoter activity was assessed by ARE Reporter kit (BPS Biosciences \#60514) combined with the dual luciferase assay (Promega \#E1910), ( \pm pre-treatment with hydrogen peroxide, $\mathrm{H}_{2} \mathrm{O}_{2} ; 250 \mu \mathrm{M}$ for $2 \mathrm{~h}$ ) [43]. Gene expression analyses were performed using identical primer pairs (Additional file 1: Table S3) and experimental parameters as described under the mouse tissues above. Immunoflourescence microscopy ( \pm pre-treatment with hydrogen peroxide, $\mathrm{H}_{2} \mathrm{O}_{2} ; 250 \mu \mathrm{M}$ for $2 \mathrm{~h}$ ) was performed after gentle fixation (4\% PFA, $10 \mathrm{~min}, 4{ }^{\circ} \mathrm{C}$ ) followed by incubation with rabbit polyclonal NRF2 antibody (Novus Bioogicals \#NBP1-32822, 1:100) and Alexa488 fluorophore conjugated goat secondary antibody (ThermoFisher \#A-11008). Cell nuclei were labelled with fluorescent DNA marker DRAQ5 (Biostatus \# DR50050) and images were acquired acquired using a Zeiss observer inverted microscope equipped with colibri 7 LED illumination, and operated using Zen (Zeiss) software.

\section{Statistics}

The data were statistically analyzed in Graphpad Prism software (version 9) and final graphs were prepared 
in Microsoft Excel. Data were analyzed by One-Way ANOVA, and pair-wise comparisons were statistically assessed by the Mann-Whitney nonparametric test or student's t-test as indicated in the respective figure legends.

\section{Results \\ IHC analyses show increased nuclear localization of phosphorylated NRF2 (p-NRF2, S40) in post-mortem PD midbrain}

A previous neuropathological study has shown that NRF2 is abundantly detected in both cytosolic and nuclear locations in the SN region, and exhibited relatively higher localization in the neuronal nuclei in PD compared to the control brains [26]. However, it remains to be determined if there is a correlation between LB pathology and nuclear enrichment of NRF2 in PD, and is potentially associated with NRF2-dependent cytoprotective and anti-oxidant response. NRF2 is highly expressed in neuronal and glial cells [44], and its stability and nuclear transcription activity are regulated by several factors including posttranslational modifications such as phosphorylation and acetylation [31]. Among the phosphoryaltion sites, serine-40 (p-S40) has been used in several studies as a surrogate marker of NRF2 stability and its nuclear localization, and remains the most widely studied post-translational modification in the published literature to date [31, 45]. Although the identification of putative kinase(s) represents an evolving field, experiments involving acute exposure of cultured cells to mitochondrial ROS inducers and site-directed mutagenesis approaches implicate a role of protein kinase C (PKC) $[29,46]$.

Accordingly, we performed IHC analyses of phosphorylated NRF2 (p-NRF2, S40) in post-mortem midbrain sections obtained from controls and PD cases (Additional file 1: Table S1). In parallel, using serial sections, we also assessed the phosphorylation of aSyn on serine residue 129 (p-aSyn, S129), which is a widely used neuropathological marker for detecting Lewy related aSyn pathology in tissue specimen $[5,37,47,48]$. Biochemically, $90 \%$ of aSyn in Lewy bodies is phosphorylated at residue S129, while this post-translational modification constitutes only $\sim 4 \%$ of aSyn in normal brain $[49,50]$.
Western immunoblotting analyses of brain homogenates from normal aged donors indicate that phosphorylated aSyn (S129) is also detected in the substantia nigra and nucleus basalis of Meynert, although its significance remains debatable [51]. Our IHC data revealed distinct neuronal nuclear p-NRF2 (S40) enrichment in PD brains compared with control brains, both in $\mathrm{SN}$ and periaqueductal grey (PAG) regions (Compare Fig. 1A, B, Controls and $1 \mathrm{C}, \mathrm{D}, \mathrm{PD}$; also see Fig. $1 \mathrm{E}, \mathrm{F}$ and Additional file 1: Fig. S1A, B). aSyn LB pathology (detected by p-aSyn, S129 IHC) was also conspicuous in SN and PAG (Fig. 1C, $1 \mathrm{E}$ and Additional file 1: Fig. S1B), and is a characteristic feature of PD $[2,4,48]$.

\section{Microarray gene expression analyses indicate aberrant NRF2 anti-oxidant response and activation of pro-apoptotic factors in PD}

Next, we investigated the functional significance of the observations concerning the increased NRF2 nuclear localization (p-NRF2, S40; Fig. 1 and Additional file 1: Fig. S1) in PD brain. In particular, we focused on assessing the expression of NRF2-responsive ROS detoxification factors (including auto-regulation), and the expression of pro-apoptotic caspases implicated in neuronal loss in PD [52]. For this purpose, we queried four publicly available transcriptomics datasets available in the GEO repository of NCBI [32-35, 39]. Following is the summary of data with noticeable differences between control and PD tissues: First, NFE2L2 (encoding NRF2) showed increased expression in in PD SN and locus coeruleus-LC with a higher (albeit, statistically not significant) expression in the dorsal motor nucleus of vagusDMX (Fig. 2A, GSE4349). Intriguingly, the expression of KEAP1 (NRF2 inhibitor) was also found to be increased in PD SN (Fig. 2B, GSE7621; Additional file 1: Fig. S3B, GSE26297), possibly suggesting compensatory NRF2Keap1 autoregulation [53] (elaborated in "Discussion" section). Second, among the NRF2 target gens involved in the anti-oxidant pathways, the expression of heme oxyganase 1 (gene symbol, HMOX1; protein, HO-1; involved in heme catabolism and ROS detoxification) was increased in PD SN and globus pallidus interna-GPi (Fig. 2C, GSE7621; GSE20146). Similarly, the expression

\footnotetext{
(See figure on next page.)

Fig. 1 Immunostaining of phospho-aSyn (S129) and phospho-NRF2 (S40) in post-mortem control and PD midbrain sections. A-D Representative IHC images showing phospho-aSyn (S129) and phospho-NRF2 (S40) immunostaining in substantia nigra (SN) and periaqueductal grey (PAG) of two controls (A-B) and four PD cases (C, D). Red arrows in the $20 \times$ magnified views point to cells with predominant nuclear localization of phospho-NRF2 (scale bar $=100 \mu \mathrm{m}$ ). Also see Additional file 1: Figure S1 showing panoramic and magnified views from a control and a PD case and Additional file 1: Table S1. Primary antibodies: p-aSyn (S129)-81A (in A, C) and p-NRF2 (S40)-EP1809Y (in B, D). E, F Semi-quantitative analyses of p-aSyn (pS129; in E) and p-NRF2 (pS40; in F) immunopositive cells in the indicated regions of control and PD midbrain sections. Individual data points represent immunopositive cell counts $/ \mathrm{mm}^{2} /$ region in each section, with controls being depicted as black triangles and PD as the black squares. (SN substantia nigra; PAG periaqueductal grey matter; One-way ANOVA: ns, not significant; ${ }^{* * *} p<0.0001$ )
} 
A) Control (p-aSyn, S129)

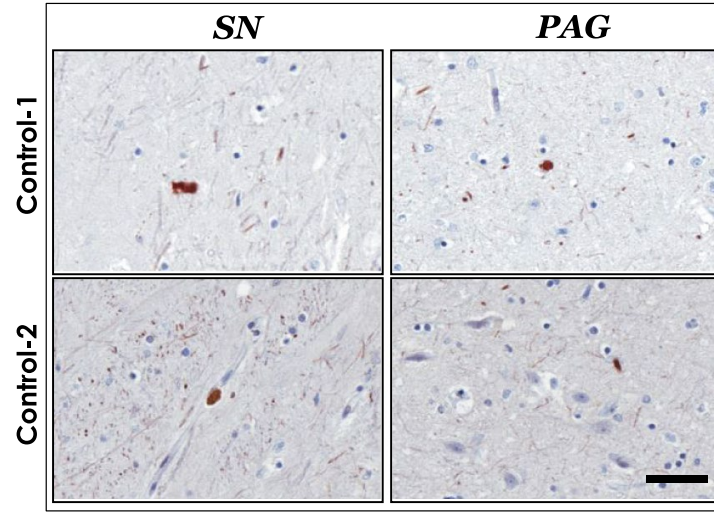

C) PD (p-aSyn, S129)

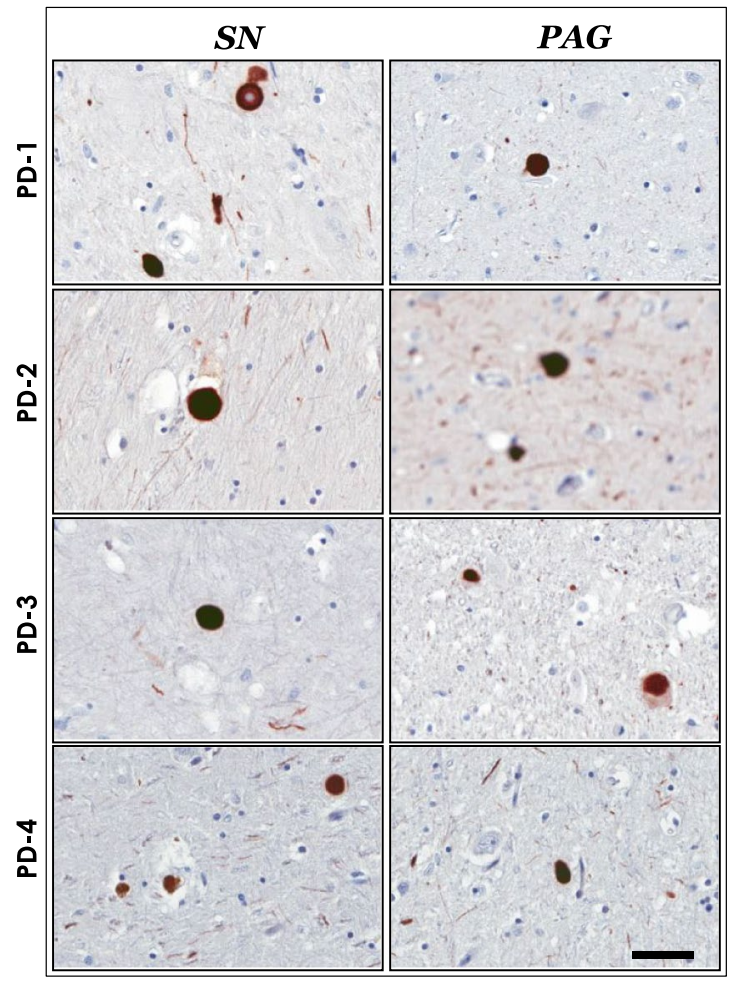

\section{E)}

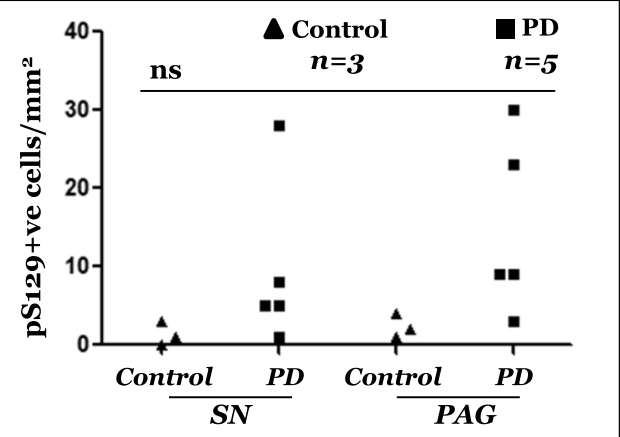

B) Control (p-NRF2, S4o)

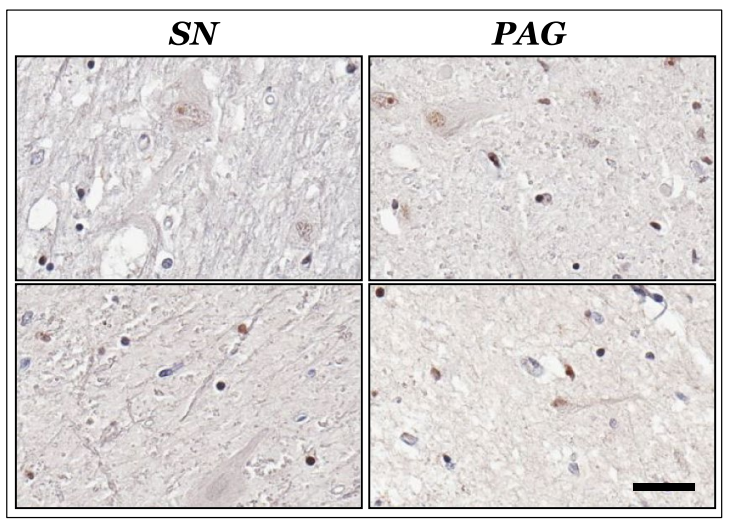

D) PD (p-NRF2, S4o $)$
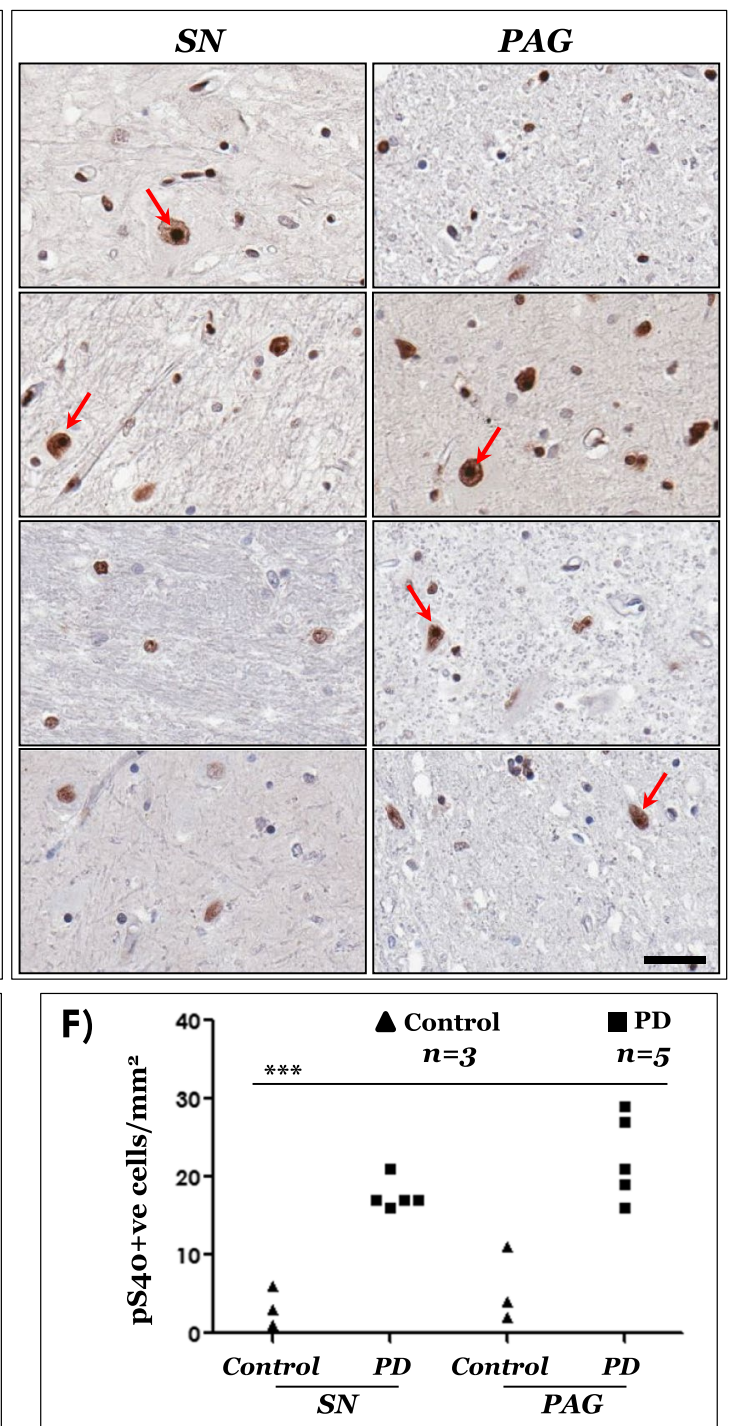


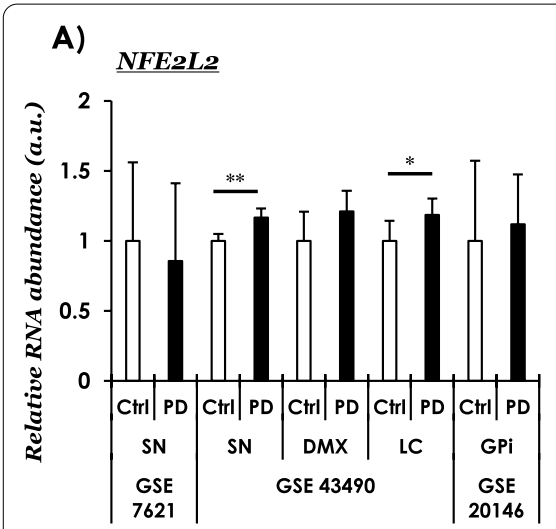

\section{B)}

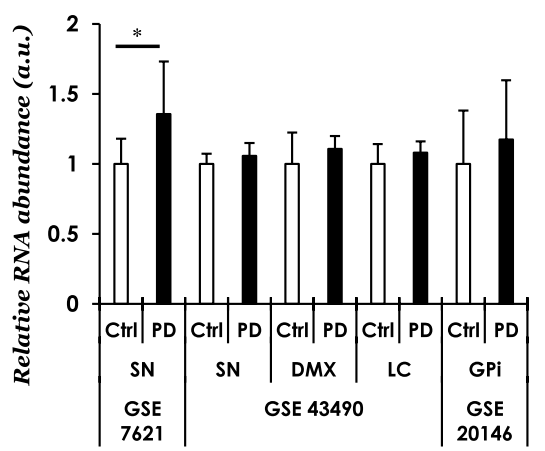

E)

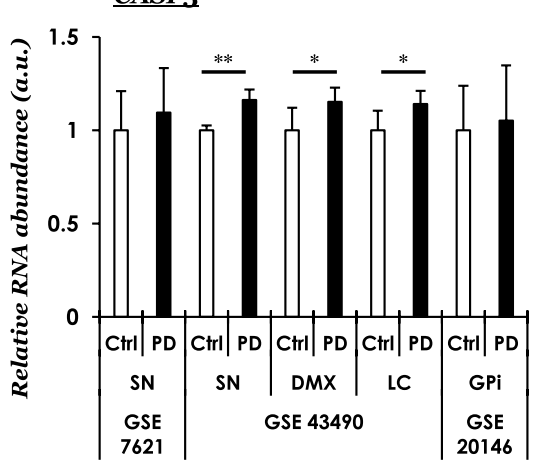

C)

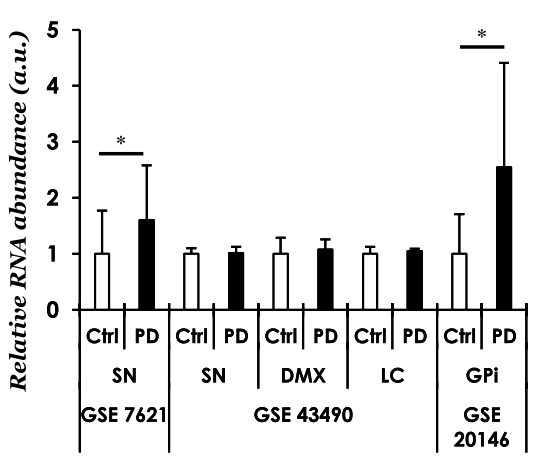

F)

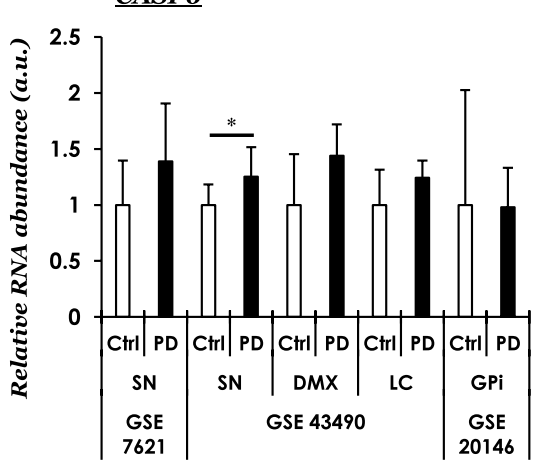

Fig. 2 Curated gene expression analyses of publicly available microarray datasets in PD using Gene Expression Omnibus (GEO). A NFE2L2 (nuclear factor, Erythroid 2 Like 2, NRF2), B KEAP1 (Kelch Like ECH Associated Protein 1, NRF2 inhibitor protein), C, D NRF2 anti-oxidant response mediators, HMOX1 (Heme Oxygenase 1, in C), GCLC (Glutamate-Cysteine Ligase Catalytic Subunit, in D), and (E, F) Pro-apoptotic caspases, CASP3 (Caspase 3, in $\mathbf{E}$ ) and CASP6 (Caspase 6, in F). The values across the datasets are expressed relative to the controls in each microarray dataset, i.e., mean value of control samples $=1$ (a.u., arbitrary units). Error bars represent standard deviation of the mean, s.d. In GSE7621-SN (substantia nigra); controls (Ctrl, $\mathrm{n}=9$ ) and PD (Parkinson Disease cases, $\mathrm{n}=16$ ); in GSE43490-SN (substantia nigra; controls, $\mathrm{n}=6$ and PD, $\mathrm{n}=8$ ), DMX (dorsal motor nucleus of vagus; controls, $n=5$ and PD, $n=8$ ), LC (locus coeruleus; controls, $n=7$ and PD, $n=8$ ); in GSE20146_GPi (globus pallidus interna; controls, $n=10$ and PD, $\mathrm{n}=10$ ). Pair-wise comparisons were assessed by Mann-Whitney test—only significant differences $\left({ }^{*}=p \leq 0.05,{ }^{* *}=p \leq 0.01\right)$ are highlighted. Probe IDs, microarray platforms and source studies are listed in Additional file 1: Table S2. Also see Additional file 1: Figure S2, S3

of gamma-glutamylcysteine synthetase (gene symbol, GCLC; a rate-limiting enzyme in the anti-oxidant glutathione synthesis) was also induced in PD SN (Fig. 2D, GSE43490). Lastly, we also probed if the local changes in NRF2 homeostatic response are potentially associated with factors regulating apoptosis, in particular caspases. Accordingly, we found slight changes in the expression of the pro-apototic caspase-3 (CASP3) in all the datasets queried; albeit, the differences were statistically significant only in GSE43490 (Fig. 2E, SN, DMX and LC). Similarly, the executioner pro-apoptotic caspase 6 (CASP6) was also found to be significantly altered in PD SN (Fig. 2F, GSE43490).

We have also probed the expression of additional NRF2-responsive genes, including $\mathrm{NAD}(\mathrm{P}) \mathrm{H}$ Quinone Dehydrogenase 1 (NQO1-involved in xenobiotic detoxification), neuroprotector molecules such as the putative NRF2 stabilizer protein deglycase DJ1 (PARK7, mutated in subset of familial PD) and inflammatory mediators interleukin-1 (IL1) and the tumor necrosis factor (TNF) (Additional file 1: Fig. S2). Among these, only NQO1 showed a significant change in PD GPi (Additional file 1: Fig. S2C, GSE20146). The dataset GSE26927 [33], shown in Additional file 1: Fig. S3, also contains the expression profiles in common neurodegenerative diseases besides PD (i.e., Alzheimer disease, Huntington disease, Motor neuron disease) in which defective ROS metabolism is implicated. Keeping in view a broad interest in the topic, we have summarized the data included in GSE26927 for readers (Additional file 1: Fig. S3); however, their elaboration is beyond the scope of this report. Collectively, these data (Fig. 2 and Additional file 1: Fig. S2) support the notion regarding altered redox homeostasis in PD. Nevertheless, it is also 
noteworthy that the expression of a given gene does not show significant differences in all the examined datasets in PD (elaborated in "Discussion" section).

\section{Brainstem a-synucleinopathy in M83 mice leads to increased phospho-NRF2 (S40) nuclear accumulation}

In order to assess the significance of pathological CNS aSyn accumulation in the context of the altered NRF2dependent gene response observed in PD, we examined phospho-NRF2 (S40) immunostaining in conjunction with the assessment of NRF2-responsive gene expression in the brains of transgenic M83 mice (expressing the aggregation prone A53T mutant human aSyn) [36]. Bilateral intramuscular inoculation of mouse PFF aSyn into the hindlimb of M83 mice results in severe morbidity and senosorimotor defects, initially characterised by a unilateral footdrop which progresses to complete paralysis 8-10 weeks post-injection [37]. The genesis of CNS aSyn neuropathology and sensorimotor deficits in this PFF based model of peripheral-to-central propagation of syncleinopathy are extensively characterized by several laboratories including our own [37, 38, 40, 54]. For these studies, we used tissues collected from terminal stage

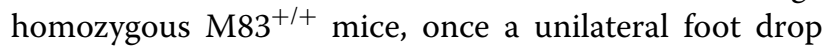
was clearly established (70-90 days post-PFF aSyn inoculation) $[37,38]$.

In line with the previous reports $[37,54]$, we also found predominant aSyn pathological affection of brainstem nuclei involved in locomotor control, and also in the periaqueductal grey (PAG). Accordingly, using immunostaining for p-aSyn (S129), we found abundant aSyn pathology in PAG, red nucleus, pontine gigantocellualr nuclei (Gi) and pontine vestibular nuclei (VN) (Fig. 3A and Additional file 1: Fig. S6A). Among the additional regions examined, aSyn (p-aSyn, S129) accumulation was relatively sparse (Fig. 3A, midbrain tegmentumcontaining $\mathrm{SN}$-and frontal cortex), or largely undetectable (Fig. 3A, cerebellar lobules $c b 1-5$ and striatum), as has also been shown [37, 54]. Additionally, p-aSyn (S129) accumulation in the brains from PBS (vehicle) injected cohort was not remarkable (Additional file 1: Fig. S4A). This is not surprising as naive M83 mice (i.e., not injected with PFF aSyn) develop spontaneous aSyn pathology usually after 8-12 months of age, and it is extremely rare before 7 months of age [36, 37].

Next, we assessed the nuclear NRF2 accumulation, by the IHC immunodetection of p-NRF2 (S40) in brain sections, from PBS and PFF aSyn injected cohorts. Robust nuclear p-NRF2 (S40) accumulation was observed in PAG, RN, Gi and VN, regions with significant p-aSyn (S129) accumulation in the PFF injected mice (Fig. 4A, also see Additional file 1: Figs. S5A and S6B, C). Furthermore, in the regions with sparse aSyn pathology, e.g., midbrain tegmentum and frontal cortex, clear distinction (i.e., nuclear vs cytosolic) of p-NRF2 (S40) immunostaining was less pronounced, compared to the aSyn pathology affected regions (Fig. 4A). Lastly, in the cerebellar nuclei (with sparse aSyn pathology), nuclear p-NRF2 (S40) immunostaining was completely lacking and/or not consistent (Fig. 4A, yellow arrows; also see Additional file 1: Fig. S6B, C). These data underline potential differences in the baseline and/or inducible degree of NRF2 response in distinct neuronal populations, and could-among other factors-represent an inherent property of their reserve for stress adaption (see "Discussion" section).

\section{a-syn pathology is associated with altered cytoprotective gene response in the brains of M83 mice.}

In a transgenic mouse model that overexpresses human (wild type) aSyn and capable of reporting an antioxidant response (via the expression of human placental alkaline phosphatase), increased expression of genes involved in ROS detoxification and glutathione biogenesis has been reported in the substantia nigra and striatum [55]. The M83 mice express the aggregation prone human mutant A53T aSyn, and develop neuroinflammation as a consequence of PFF induced pathological aSyn accumulation in the CNS $[37,54]$. Therefore, we wanted to determine

\footnotetext{
(See figure on next page.)

Fig. 3 Immunostaining of phospho-aSyn (S129) in the brain regions of PFF aSyn injected M83 ${ }^{+/+}$mice. A Representative IHC images showing phospho-aSyn (S129) immunostaining in neuronal somata and processes in the indicated brain regions. Also, notice the scarcity of staining in cerebellar lobules cb1-5 (purkinje and granule cells-yellow arrow in the inset), motor cortex and corpus striatum (10 $\times$ low magnification views and $40 \times$ magnified views in the insets; scale bar $=200 \mu \mathrm{m}$; Aq. in the image showing PAG, cerebral aqueduct; $4 \mathrm{v}$. in the image showing vestibular nuclei image, 4th ventricle). Bregma co-ordinates for the brain regions were determined, according to Paxinos and Franklin: (Bregma, - $3.87 \mathrm{~mm}$ ) midbrain at the level of superior colliculi showing PAG, red nucleus and tegmentum; (Bregma, -5.99$)$ pontocerebellar junction showing cerebellar nuclei, cerebellar lobules (cb1-5), vestibular nuclei and pontine gigantocellualr nuclei (Gi); and (Bregma, 0.49 mm) forebrain showing motor cortex (M1 and M2) and corpus striatum). Also see Additional file 1: Fig. S4A (PBS injected cohort) and Additional file 1: Fig. S6A (additional high resolution data from the PFF injected cohort). Primary antibody in A: p-aSyn (S129)_-11A5. B Semi-quantitative analyses of p-aSyn (pS129) immunopositive

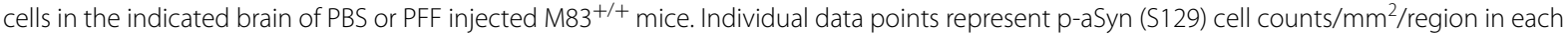
animal of the respective cohort, with PBS cohort being depicted as blank triangles and PFF as the black squares. (PAG periaqueductal grey matter; $R N$ red nucleus; Gi pontine gigantocellualr nuclei; VN pontine vestibular nuclei; One-way ANOVA, $\left.{ }^{* * *} p<0.0001 ; n=5 / g r o u p\right)$
} 


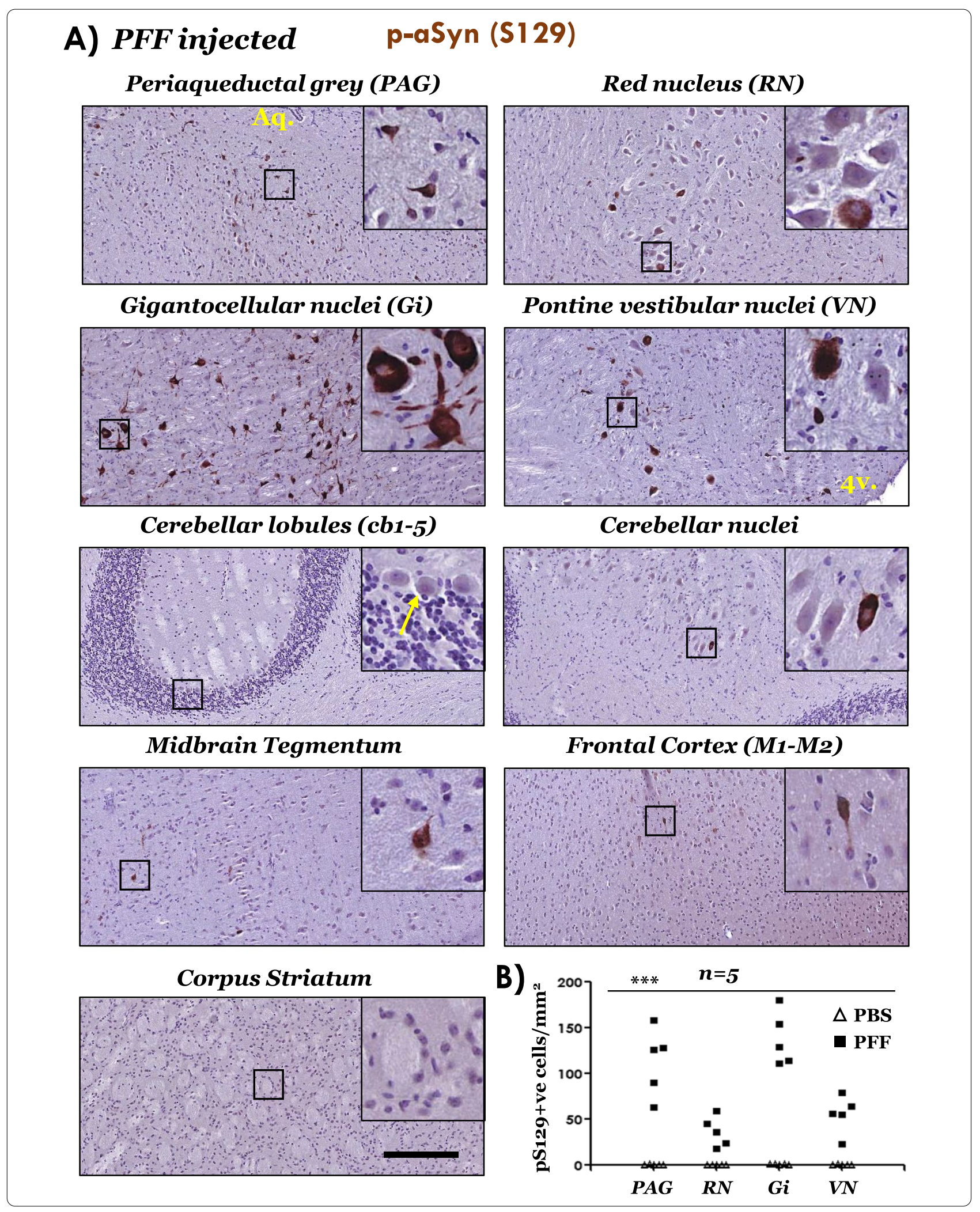


if the changes in nuclear localization of NRF2 (p-NRF2, S40) in the brains regions harboring pathological aSyn accumulation (i.e., p-S129) in the M83 PFF model leads to NRF2-dependent tissue response, i.e., the expression of anti-oxidant and cytoprotective genes. For this purpose, we assessed gene expression (by qRT-PCR) across the brain neuraxis, both in regions harboring significant aSyn pathology (i.e., brainstem), as well as in the regions which were largely unaffected (e.g., hippocampus, striatum). Our data show that the expression of NRF2 mRNA (Nfe2l2) was relatively unaltered between PBS and PFF injected mice (Additional file 1: Fig. S7A). Remarkably, the expression of NRF2-inhibitor (Keap1) was significantly increased in frontal cortex and in the pons region of PFF-injected mice (Fig. 5A), which potentially hints to region specific anti-oxidant response regulation (see "Discussion" section). Among the anti-oxidant genes, the expression levels of three important ROS scavangers, Hmox1, Gclc and Gsr (protein: Glutathione S-Reductase-reduces oxidized glutathione disulfide to the anti-oxidant form of glutathione-) were significantly increased in the PFF-injected cohort (Fig. 5B-D). Among other NRF2-related pathways, the changes were either inconsistent (Additional file 1: Fig. S7B; Txn, protein: thioredoxin - an anti-oxidant factor in response to intracellular nitric oxide, also inhibits caspase- 3 activity), or were not significant (Additional file 1: Fig. S7C, Nqo 1 and S7D, Park7).

In tandem, we also assessed the effects of altered NRF2-anti-oxidant activity on pro-apoptotic and neuroinflammatory pathways, since neuroinflammation has been implicated in PD pathogenesis [18], and is also a characteristic feature in the brains of terminal stage PFF M83 mouse model [37, 54]. We found that the expression of mRNAs encoding pro-inflammatory mediators, namely Tnf- $a$ (protein, tumor necrosis factor- $\alpha$ ) and $I l 1-a$ (proprotein, interleukin-1 $\alpha$ ) was significantly elevated in pons and midbrain of PFF-injected M83 mice (Additional file 1: Fig. S8A, B, respectively). In contrast, the expression of pro-apoptotic effector caspases was relatively unaltered, except localized changes in Casp3 (Additional file 1: Fig. S8C, pons) and Casp6 (Additional file 1: Fig. $\mathrm{S} 8 \mathrm{C}$, midbrain) in the PFF-injected mice. Hence, the collective tissue response in the brainstem regions-consisting of an increased p NRF2 (S40) nuclear localization (Fig. 4 and Additional file 1: Fig. S6) and corresponding anti-oxidant gene expression (Fig. 5B, D)-supports the notion that NRF2 homeostatic response is influenced by pathological p-aSyn (p-S129) accumulation.

\section{Transient aSyn overexpression is not a potent stimulus to activate NRF2 anti-oxidant response in cultured N2A cells}

We and others have reported that exogenous application of aggregated aSyn and/or transient aSyn overexpression is associated with altered cellular phenotype including oxidative stress and altered cellular respiration $[38,56,57]$. Therefore, we wanted to investigate if acute (short-term) intracellular aSyn accumulation also activates NRF2 anti-oxidant activity and compensatory gene response. Our data show that transient overexpression of wild type or mutant human A53T aSyn in cultured neuroblastoma N2A cells did not alter cellular NRF2 expression (Additional file 1: Fig. S9A, B), and was associated with only slightly increased ROS accumulation (Additional file 1: Fig. S9C; compare with hydrogen peroxide$\mathrm{H}_{2} \mathrm{O}_{2}$ treated groups). In addition, aSyn overexpressing cells did not exhibit a robust anti-oxidant response element (ARE) NRF2 activity compared with the mock transfected cells (Additional file 1: Fig. S9D; also compare with hydrogen peroxide- $\mathrm{H}_{2} \mathrm{O}_{2}$ treated groups). Accordingly, there were non-significant changes in the NRF2depdendent gene expression, i.e., Nf2l2, Keap1, Gclc, Gsr 1 and Hmox1 (Additional file 1: Fig. S9E), which are characteristically activated in this experimental cell model by the application of exogenous ROS inducers [43]. Lastly, using immunofluorescence microscopy, we detected NRF2 predominantly in diffuse cytoplasmic localization in the cells, with sparse detection of nuclear NRF2 in mock, WT aSyn or A53T aSyn groups (Additional file 1:

\footnotetext{
(See figure on next page.)

Fig. 4 Immunostaining of phospho-NRF2 (S40) in the brain regions of PFF aSyn injected M83 ${ }^{+/+}$mice. A Representative IHC images showing phospho-NRF2 (S40) immunostaining with distinct nuclear staining in brainstem regions (red arrows). Also, notice the predominantly cytoplasmic staining in cerebellar lobules (purkinje cells), motor cortex and corpus striatum-yellow arrows $(10 \times$ low magnification views and $40 \times$ magnified views in the insets; scale bar $=200 \mu \mathrm{m}$; Aq. in the image showing PAG, cerebral aqueduct; $4 \mathrm{v}$. in the image showing vestibular nuclei image, 4th ventricle). Bregma co-ordinates for the brain regions were determined, according to Paxinos and Franklin: (Bregma, $-3.63 \mathrm{~mm}$ ) midbrain at the level of superior colliculi showing PAG, red nucleus and tegmentum; (Bregma, -5.79) pontocerebellar junction showing cerebellar nuclei, cerebellar lobules (cb1-5), vestibular nuclei and pontine gigantocellualr nuclei (Gi); and (Bregma, $0.73 \mathrm{~mm}$ ) forebrain showing motor cortex (M1 and M2) and corpus striatum. Also see Additional file 1: Fig. S5A (PBS injected cohort) and Additional file 1: Fig. S6B, C (additional high resolution data from the PFF and PBS cohorts). Primary antibody in A: p-NRF2 (S40)-PA5-67520. B Semi-quantitative analyses of p-NRF2 (pS40) immunopositive cells in the

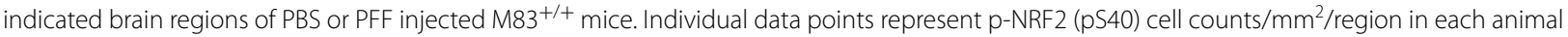
of the respective cohort, with PBS cohort being depicted as blank triangles and PFF as the black squares. (PAG periaqueductal grey matter; $R N$ red nucleus; Gi pontine gigantocellualr nuclei; VN pontine vestibular nuclei; one-way ANOVA, ${ }^{* * *} p<0.0001 ; n=5 /$ group)
} 


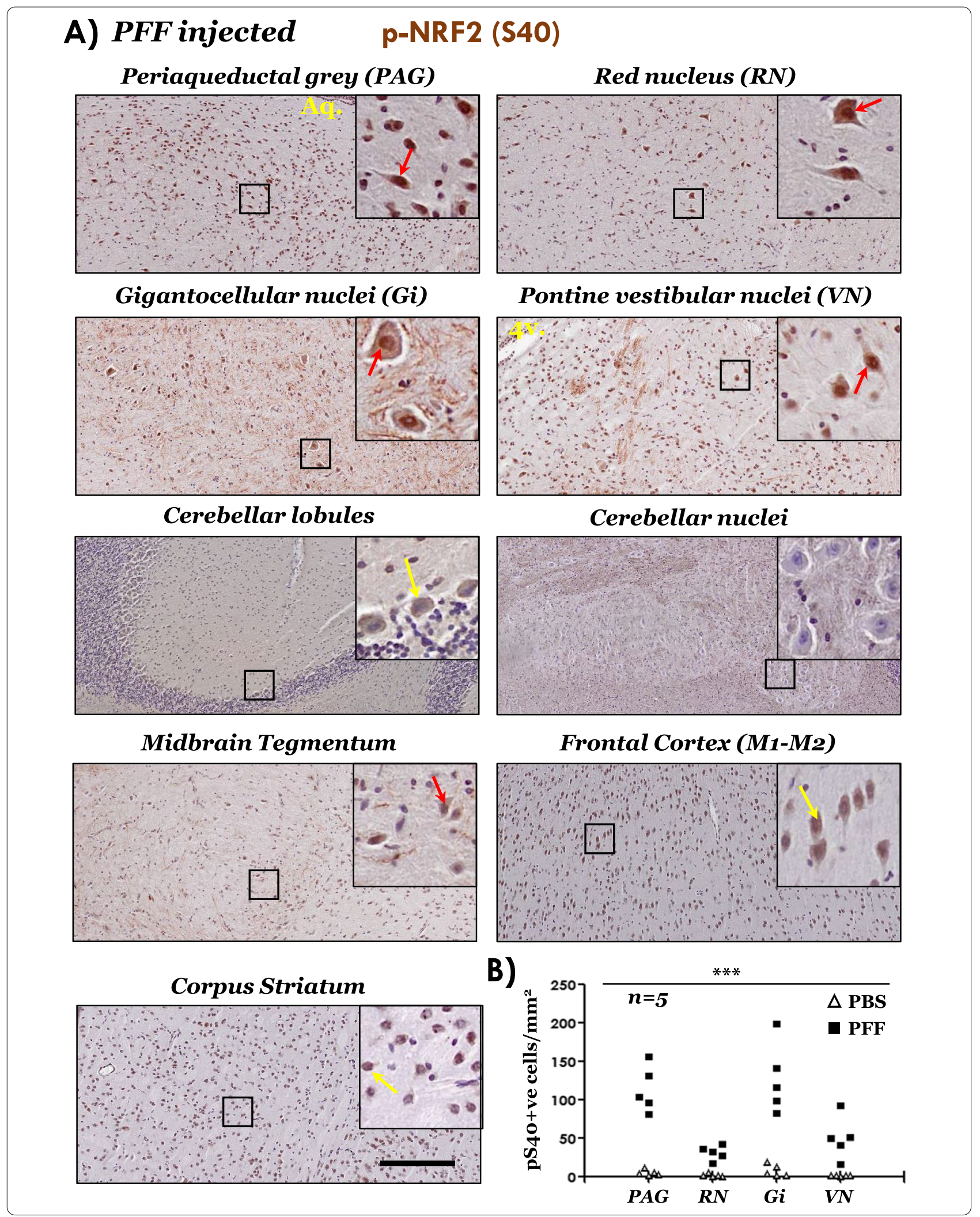



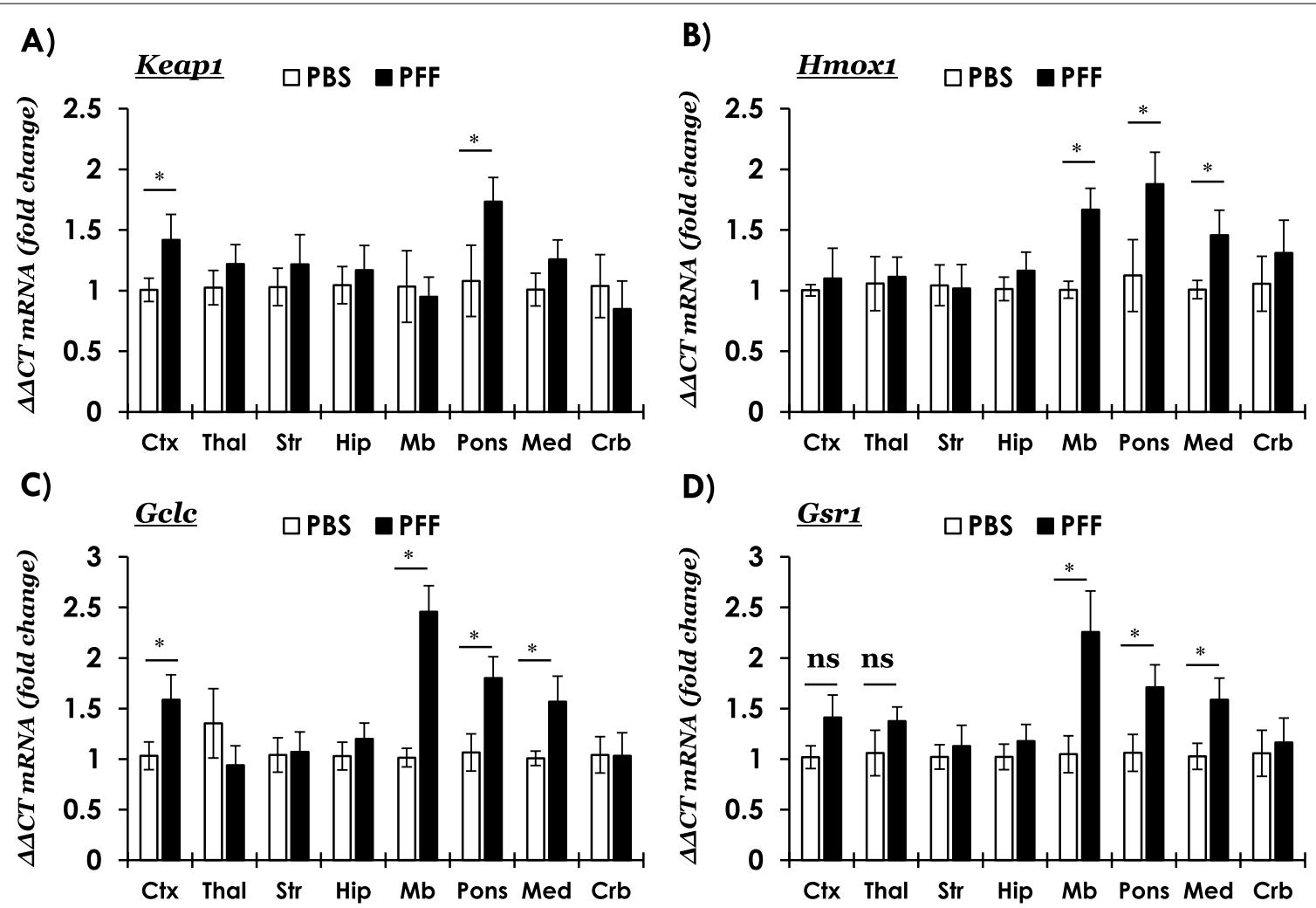

Fig. 5 Quantitative RT-PCR analyses of anti-oxidant gene response in the brain regions of PBS or PFF aSyn injected M83 ${ }^{+/+}$mice. A Keap 1 (Kelch Like ECH Associated Protein 1, NRF2 inhibitor protein), and B-D NRF2 anti-oxidant response mediators, Hmox 1 (Heme Oxygenase 1, in B, Gclc (glutamate-cysteine ligase catalytic subunit, in C and Gsrl (glutathione-disulfide reductase, in D. Relative mRNA quantitation at terminal stage in the following brain regions: frontal cortex (Ctx), thalamus (Thal), corpus striatum (Str), hippocampus (Hip), midbrain (Mb) pons, medulla (Med) and cerebellum (Crb). Mouse Gapdh was used as a reference gene ( $\triangle \Delta C T$ method: $n=6 /$ group, samples were run in duplicates; Error bars represent standard error of the mean, s.e.m.; Pair-wise comparisons were assessed by Mann-Whitney test-only significant differences $\left({ }^{*}=p \leq 0.05\right)$ are highlighted (ns=not significant); Multiple columns comparisons in One way ANOVA post-hoc Bonferroni test were not significant). Also see Additional file 1: Fig. S7

Fig. S9F; compare with $\mathrm{S} 9 \mathrm{G}-\mathrm{H}_{2} \mathrm{O}_{2}$ treated groups). These data indicate that the acute (short-term) phase of intracellular aSyn accumulation in this cellular model is not a robust stimulus to activate NRF2, and potentially additional mechanisms may be required for non-physiological aSyn accumulation to mediate these pathways (elaborated in "Discussion" section).

\section{Discussion}

PD represents a multifactorial neurological disorder with underlying role of several genetic risk factors, and is defined by distinct neuropathological subtypes $[1,2,4]$. Furthermore, given the complex etiology of PD, it is likely that neuronal dysfunction and demise result from interplay of several factors, including genetic and environmental influences, cellular adaptations to the deleterious effects of mutations increasing disease risk, and neuronal reserve to mitigate metabolic challenges incurred by proteopathic stress $[1,2,12]$. Based on the genetics and pathology of PD, significant research efforts have focused on elucidating the consequences of neuronal aSyn accumulation, which could potentially guide the discovery of mechanisms relevant to neurodegeneration [11]. In this context, considerable evidence points to the cytotoxic effects of aSyn aggregation, and a prion-like behavior during propagation in the nervous system $[4,9]$. In particular, several studies point to the deleterious effects of cellular aSyn accumulation on mitochondrial function and impaired energy homeostasis [13, 18]. In broader terms, it is plausible that augmenting the activity of cellular mediators promoting mitochondrial function and/ or mitigating the deleterious effects of redox imbalance could have a therapeutic potential in PD and related diseases $[12,16]$.

In this report we show that in PD midbrain harboring pathological LB lesions (SN and PAG), there is significant nuclear enrichment of phosphorylated NRF2 (S40), a post-translational modification putatively indicating 
augmented NRF2 dependent anti-oxidant response (Fig. 1D, 1F and Additional file 1: Fig. S1B). This is supported by the curated assessment of NRF2-responsive gene expression in independent microarray studies examining disease affected regions in PD (SN, DMX, LC and GPi). Among the examined anti-oxidant genes, analyses of two microarray studies indicate significant changes in the expression of Heme oxygenase, HO-1 (HMOX1) in PD SN and GPi (Fig. 2C), which was also reflected in the brainstem of end-stage $\mathrm{M} 3^{+/+}$mice (Fig. 5B). These observations are in line with biochemical reports showing higher concentrations of HO-1 in the serum of PD patients [58] and in PD brain homogenates [59], as well as increased HO-1 histopathological localization in the LB inclusions [60] and in glial cells (astrocytes and microglia) within PD SN [59]. We also found relatively increased expression of NQO1 (encoding a reductase in the xenobiotic detoxification pathway) in the PD GPi (Additional file 1: Fig. S2C, GSE20146), although the expression data in the PD SN were not statistically significant (Additional file 1: Fig. S2C, GSE7621). Interestingly, NQO1 protein is sequestered in LB inclusion in the PD SN, thus further supporting a role of impaired NRF2 dependent cytoprotection in PD [61].

To a considerable extent, the IHC findings in the postmortem PD midbrain (Fig. 1), and in the brains of endstage M83 mice (Figs. 3, 4) implicate pathological aSyn deposition and/or aggregation as a trigger for altered p-NRF2 localization. However, it is plausible that the accumulation of phosphorylated aSyn (p-aSyn, S129) and the nuclear enrichment of p-NRF2 are parallel events. A compelling case linking the two processes can be made by further systematic analyses, for instance analysing the nuclear NRF2 accumulation in PD cases at different neuropathological stages, and also in brain regions relatively spared by pathological aSyn accumulation (with inclusion of appropriate controls), and possibly in other synucleinopathies (MSA, DLB). An alternative, and more feasible, line of evidence would be to examine the relationship of pathological aSyn accumulation and nuclear NRF2 in the PFF M83 or other synucleinopathy models at early and late stages of pathology progression across the affected neuraxis [54]. Another tantalizing area for future studies could be to investigate whether misfolded aSyn directly interacts with NRF2, factors controlling NRF2 nuclear accumulation, and/or if such hypothetical interactions affect the NRF2 anti-oxidant response. In this regard, our studies in cultures of dopaminergic $\mathrm{N} 2 \mathrm{~A}$ cells indicate that ectopic (plasmid) overexpression of aSyn (WT or A53T mutant) was not sufficient to trigger a potent NRF2 nuclear translocation or compensatory gene expression (Additional file 1: Fig. S9). These data potentially hint towards the role of aSyn aggregation
[56, 57], and the effect of post-translational modifications and/or aSyn truncations characteristically found in disease affected tissues that may alter aSyn protein-protein interactions [11]. Conversely, studies using neuronal cultures show that NRF2 accelerates the clearance of aSyn, shortening its half-life and decreases the overall aSyn expression levels through yet to decipher mechanism(s) [62].

Two sets of observations in these data to certain extent are intriguing, and remain largely unexplained: First, the expression profile of the genes examined (e.g., $H M O X 1$, $C A S P 3)$ was not uniformly altered across the PD microarray datasets (Fig. 2, Additional file 1: Fig. S2-S3). This could potentially reflect heterogeneity in the cohorts (e.g., stage of pathology, extent of neurodegeneration, treatment regimen etc.). Second, the expression of NRF2 inhibitor Keap1 was paradoxically increased in the presence of pathological aSyn accumulation, i.e., PD SN (Fig. 2A; GSE7621) and pons of M83 mice (Fig. 5A). In this regard, post-mortem IHC analyses in PD show that Keap1 is localized in the Lewy bodies in brainstem and substantia nigra, along with p62 protein (Sequestome 1, a multifunctional protein and marker of intracellular inclusion formation) [63]. It has been suggested that p62 phosphorylation increases the sequestration of Keap1 into inclusions, thus facilitating NRF2-Keap1 dissociation and activation of NRF2 dependent gene response in PD and other neurodegenerative diseases [64]. Furthermore, cell cultures studies also hint towards NRF2 mediated autoregulation via altered Keap1 expression and/or activity [53], and other ancillary mechanisms [23, 31]. Moreover, given the putative regulatory role of NRF2 homeostatic response in ROS independent metabolic regulation (e.g., glutamine biogenesis, signal transduction) [23, 31, 65], these data also hint towards distinct compensatory mechanism(s) that could be a particular feature of different neuronal populations in a region (e.g., neurotransmitter phenotype, metabolic demands and adaptive response to stress). Specifically, the cellular difference in inducible NRF2 expression could play a significant role, as reflected by the studies showing that astrocytic NRF2 activation influences the redox homeostasis in neurons, and is a crucial factor in neuroprotection under oxidative stress [44, 66, 67]. Lastly, it is also plausible that the increased p-NRF2 (S40) nuclear immunostaining reflects a decreased nuclear export of NRF2 as a result of altered metabolic signaling in additional pathways (e.g., glycogen synthase kinase 3 $\beta$, Fyn) [31].

Several studies in cultured cells and animal models indicate that misfolded and/or aggregated aSyn triggers a cytoprotective NRF2 response [55, 59, 61, 62], and conversely NRF2/Keap1 pathway plays a pivotal role in improving neuronal survival against aSyn aggregation 
and toxicity [62, 64, 68]. For instance, ectopic expression of human aSyn (via recombinant adeno-associated vector-rAAV delivery) in the SN of NRF2 knockout mice promotes aSyn aggregation, and aggravates the loss of dopaminergic neurons in association with pro-inflammatory gliosis [59]. Similarly, exposure to oligomeric aSyn leads to reduced expression of anti-oxidants Hmox 1 and $\mathrm{NqO} 1$ and increased expression of pro-inflammatory $I l 1 b$, Il6 and iNos expression in cultured microglia and astrocytes lacking NRF2 [59]. Furthermore, the aggregation prone human A53T mutant aSyn exposure triggers pro-inflammatory microglial activation, putatively via mechanisms involving mitogen activated protein kinases (MAPKs) [69]. From a therapeutic perspective, several small molecules activators of NRF2 pathway restore mitochondrial function under conditions of redox stress, both in cell cultures and also in models of neurodegenerative diseases $[25,28,70]$. For instance, NRF2 activating electrophile tert-butylhydroquinone ( $\mathrm{tBHQ}$ ) reduces oxidative stress and mitigates mitochondrial deficits in N2A cells transiently transfected with A53T mutant aSyn [68]. Importantly, a therapeutic molecule, dimethyl fumarate (DMF, Tecfidera), has been successfully used in relapsing-remitting cases of multiple sclerosis, putatively owing to its anti-inflammatory and anti-oxidant effects [71]. In cultures of dopaminergic MN9D cells, short-term (4-24 h) DMF exposure modulates autophagic flux and increases the expression of anti-oxidant HO-1 [61]. Furthermore, treatment with DMF, or its active metabolite monomethyl fumarate (MMF), has also been shown to be associated with beneficial outcomes in a number of PD animal models based on (transgenic or viral) aSyn overexpression or chemically induced Parkinsonism [71]. These studies show that pharmacological NRF2 activation reduces oxidative stress, prevents neuronal loss, with a concomitant decrease in pathological p-aSyn (S129) accumulation [71]. Oral DMF delivery in mice expressing human aSyn in SN (via rAAV injection) is also reported to be associated with reduced loss of dopaminergic neurons, improved motor performance and prevention of pro-inflammatory gliosis [61]. Similarly, increasing NRF2 expression or promoting nuclear localization (by KEAP1 downregulation) rescues the loss of dopaminergic neurons, and improves locomotor performance in PD Drosophila models [72, 73].

We have previously reported that modulating the activity of a calcium calmodulin eukaryotic elongation factor-2 kinase (eEF2K) exerts a regulatory influence on NRF2 nuclear localization and anti-oxidant response in an ROS-independent manner [43]. We showed that eEF2K knockdown improved mitochondrial respiration and ROS scavenging capability of cultured dopaminergic neurons, as well as improved locomotor performance of C. elegans expressing human A53T mutant aSyn or human amyloid- $\beta 42[38,43]$. Notably, we also reported that eEF2K (mRNA) expression and/or activity is pathologically increased in post-mortem PD and Alzheimer disease brains, as well as in relevant transgenic rodent models including the moribund $\mathrm{M} 83^{+/+}$mice $[38,43]$. This highly conserved regulatory pathway plays a crucial role in controlling protein synthesis (an energy demanding process), and is also implicated in synaptic function $[74,75]$. Pharmacological eEF2K inhibition remains an active area of interest for therapeutic discovery in oncology [76]; however, the therapeutic potential of this approach in neurodegenerative diseases remains largely untapped. In conclusion, taken together with several predecessor studies, our data provide further supporting evidence to highlight the role of NRF2/Keap1 pathway in $\mathrm{PD}$, and underscores the relevance of modulating the activity of this pathway for potential therapies in PD and related diseases.

\section{Supplementary Information}

The online version contains supplementary material available at https://doi. org/10.1186/s40478-021-01209-3.

Additional file 1. Supplementary Information, Delaidelli A. et al.

\section{Acknowledgements}

The authors would like to thank Helene Andersen (JRN lab) and Sandra Bonnesen (CV) for technical help during the study.

\section{Authors' contributions}

$A D, M R, L J, N F$ and AJ designed research; AD, MR, LJ, AVDL, IBJC, NF and AJ performed research; IM provided human post-mortem tissue and contributed to discussion; CBV, PHJ, JRN and PHS contributed with infrastructural support, data evaluation and discussion; $A D$ and $A J$ wrote the manuscript. All the authors read and approved the manuscript. $A D$ and MR contributed equally.

\section{Funding}

This work was supported by funding to AJ in the form of a Marie Skłodowska Curie Fellowship from European Union's Horizon 2020 Research and Innovation Programme (MSCA-IF-2017, Grant \#786433) and the Lundbeckfonden, Denmark (Grant \#R250-2017-1131), NF was supported by the Lundbeckfonden (Grant \#R171-2014-591), MR was funded by Aarhus Universitets Forskningsfond starting grant (Grant \# AUFF-E-2015-FLS-8-4) and JNR was supported by the Henny Sophie Clausen og møbelarkitekt Aksel Clausens Fond.

\section{Availability of data and materials}

The transcriptomic datasets analyzed during this study can be accessed on the NCBI GEO webpage [39], with the accession and probe IDs provided in Additional file 1: Table S2. Otherwise, all the data generated and analyzed during this study are included in the main manuscript or the associated supplementary files.

\section{Declarations}

Competing interests

The authors declare no competing interests.

\section{Author details}

${ }^{1}$ Danish Research Institute of Translational Neuroscience (DANDRITE)- Nordic-EMBL Partnership for Molecular Medicine, Department of Biomedicine, 
Aarhus University, Ole Worms Allé 3, 8000 Aarhus C, Denmark. ${ }^{2}$ Core Center for Molecular Morphology, Section for Stereology and Microscopy Department of Clinical Medicine, Aarhus University Hospital, 8200 Aarhus N, Denmark. ${ }^{3}$ Department of Pathology and Laboratory Medicine, University of British Columbia, Vancouver V6T 2B5, Canada. ${ }^{4}$ British Columbia Cancer Research Centre, Vancouver, BC V5Z 1L3, Canada.

Received: 2 May 2021 Accepted: 29 May 2021

Published online: 06 June 2021

\section{References}

1. Kalia LV, Lang AE (2015) Parkinson's disease. Lancet 386:896-912. https://doi. org/10.1016/S0140-6736(14)61393-3

2. Poewe W, Seppi K, Tanner CM, Halliday GM, Brundin P, Volkmann J et al (2017) Parkinson disease. Nat Rev Dis Primers 3:17013. https://doi.org/10.1038/nrdp. 2017.13

3. Schapira AHV, Chaudhuri KR, Jenner P (2017) Non-motor features of Parkinson disease. Nat Rev Neurosci 18:509. https://doi.org/10.1038/nrn.2017.91

4. Braak H, Del Tredici K, Rub U, de Vos RA, Jansen Steur EN, Braak E (2003) Staging of brain pathology related to sporadic Parkinson's disease. Neurobiol Aging 24:197-211

5. Goedert M, Jakes R, Spillantini MG (2017) The synucleinopathies: twenty years on. J Parkinsons Dis 7:S51-S69. https://doi.org/10.3233/JPD-179005

6. Polymeropoulos MH, Lavedan C, Leroy E, Ide SE, Dehejia A, Dutra A et al (1997) Mutation in the alpha-synuclein gene identified in families with Parkinson's disease. Science 276:2045-2047

7. Spillantini MG, Schmidt ML, Lee VM, Trojanowski JQ, Jakes R, Goedert M (1997) Alpha-synuclein in Lewy bodies. Nature 388:839-840. https://doi.org/ $10.1038 / 42166$

8. Braak H, Rub U, Sandmann-Keil D, Gai WP, de Vos RA, Jansen Steur EN et al (2000) Parkinson's disease: affection of brain stem nuclei controlling premotor and motor neurons of the somatomotor system. Acta Neuropathol 99:489-495. https://doi.org/10.1007/s004010051150

9. Uchihara T, Giasson BI (2016) Propagation of alpha-synuclein pathology: hypotheses, discoveries, and yet unresolved questions from experimental and human brain studies. Acta Neuropathol 131:49-73. https://doi.org/10. 1007/s00401-015-1485-1

10. Bose A, Beal MF (2016) Mitochondrial dysfunction in Parkinson's disease. J Neurochem 139(Suppl 1):216-231. https://doi.org/10.1111/jnc.13731

11. Lashuel HA, Overk CR, Oueslati A, Masliah E (2013) The many faces of alphasynuclein: from structure and toxicity to therapeutic target. Nat Rev Neurosci 14:38-48. https://doi.org/10.1038/nrn3406

12. Schapira AH (2008) Mitochondria in the aetiology and pathogenesis of Parkinson's disease. Lancet Neurol 7:97-109. https://doi.org/10.1016/S14744422(07)70327-7

13. Wong YC, Krainc D (2017) alpha-synuclein toxicity in neurodegeneration: mechanism and therapeutic strategies. Nat Med 23:1-13. https://doi.org/10. 1038/nm.4269

14. Orenstein SJ, Kuo SH, Tasset I, Arias E, Koga H, Fernandez-Carasa I et al (2013) Interplay of LRRK2 with chaperone-mediated autophagy. Nat Neurosci 16:394-406. https://doi.org/10.1038/nn.3350

15. Tolosa E, Vila M, Klein C, Rascol O (2020) LRRK2 in Parkinson disease: challenges of clinical trials. Nat Rev Neurol 16:97-107. https://doi.org/10.1038/ s41582-019-0301-2

16. Henchcliffe C, Beal MF (2008) Mitochondrial biology and oxidative stress in Parkinson disease pathogenesis. Nat Clin Pract Neurol 4:600-609. https://doi. org/10.1038/ncpneuro0924

17. Schapira AH, Cooper JM, Dexter D, Jenner P, Clark JB, Marsden CD, (1989) Mitochondrial complex I deficiency in Parkinson's disease. Lancet 1:1269

18. Beal MF (2003) Mitochondria, oxidative damage, and inflammation in Parkinson's disease. Ann NY Acad Sci 991:120-131. https://doi.org/10.1111/j. 1749-6632.2003.tb07470.x

19. Dexter DT, Carter CJ, Wells FR, Javoy-Agid F, Agid Y, Lees A et al (1989) Basal lipid peroxidation in substantia nigra is increased in Parkinson's disease. J Neurochem 52:381-389. https://doi.org/10.1111/j.1471-4159.1989.tb09133.x

20. Scudamore O, Ciossek T (2018) Increased oxidative stress exacerbates alpha-synuclein aggregation in vivo. J Neuropathol Exp Neurol 77:443453. https://doi.org/10.1093/jnen/nly024
21. Johnson JA, Johnson DA, Kraft AD, Calkins MJ, Jakel RJ, Vargas MR et al (2008) The Nrf2-ARE pathway: an indicator and modulator of oxidative stress in neurodegeneration. Ann N Y Acad Sci 1147:61-69. https://doi. org/10.1196/annals.1427.036

22. Mitsuishi Y, Motohashi H, Yamamoto M (2012) The Keap1-Nrf2 system in cancers: stress response and anabolic metabolism. Front Oncol 2:200. https://doi.org/10.3389/fonc.2012.00200

23. Bryan HK, Olayanju A, Goldring CE, Park BK (2013) The Nrf2 cell defence pathway: Keap1-dependent and-independent mechanisms of regulation. Biochem Pharmacol 85:705-717. https://doi.org/10.1016/j.bcp.2012. 11.016

24. Paladino S, Conte A, Caggiano R, Pierantoni GM, Faraonio R (2018) Nrf2 pathway in age-related neurological disorders: insights into MicroRNAs. Cell Physiol Biochem 47:1951-1976. https://doi.org/10.1159/000491465

25. Calkins MJ, Johnson DA, Townsend JA, Vargas MR, Dowell JA, Williamson TP et al (2009) The Nrf2/ARE pathway as a potential therapeutic target in neurodegenerative disease. Antioxid Redox Signal 11:497-508. https:// doi.org/10.1089/ARS.2008.2242

26. Ramsey CP, Glass CA, Montgomery MB, Lindl KA, Ritson GP, Chia LA et al (2007) Expression of Nrf2 in neurodegenerative diseases. J Neuropathol Exp Neurol 66:75-85. https://doi.org/10.1097/nen.0b013e31802d6da9

27. Suh JH, Shenvi SV, Dixon BM, Liu H, Jaiswal AK, Liu RM et al (2004) Decline in transcriptional activity of Nrf2 causes age-related loss of glutathione synthesis, which is reversible with lipoic acid. Proc Natl Acad Sci U S A 101:3381-3386. https://doi.org/10.1073/pnas.0400282101

28. Todorovic M, Wood SA, Mellick GD (2016) Nrf2: a modulator of Parkinson's disease? J Neural Transm (Vienna) 123:611-619. https://doi.org/10.1007/ s00702-016-1563-0

29. Huang HC, Nguyen T, Pickett CB (2002) Phosphorylation of Nrf2 at Ser-40 by protein kinase $C$ regulates antioxidant response element-mediated transcription. J Biol Chem 277:42769-42774. https://doi.org/10.1074/jbc. M206911200

30. Niture SK, Jain AK, Jaiswal AK (2009) Antioxidant-induced modification of INrf2 cysteine 151 and PKC-delta-mediated phosphorylation of Nrf2 serine 40 are both required for stabilization and nuclear translocation of Nrf2 and increased drug resistance. J Cell Sci 122:4452-4464. https://doi. org/10.1242/jcs.058537

31. Niture SK, Khatri R, Jaiswal AK (2014) Regulation of Nrf2-an update. Free Radic Biol Med 66:36-44. https://doi.org/10.1016/j.freeradbiomed.2013. 02.008

32. Corradini BR, lamashita P, Tampellini E, Farfel JM, Grinberg LT, MoreiraFilho CA (2014) Complex network-driven view of genomic mechanisms underlying Parkinson's disease: analyses in dorsal motor vagal nucleus, locus coeruleus, and substantia nigra. Biomed Res Int 2014:543673. https://doi.org/10.1155/2014/543673

33. Durrenberger PF, Fernando FS, Kashefi SN, Bonnert TP, Seilhean D, NaitOumesmar B et al (2015) Common mechanisms in neurodegeneration and neuroinflammation: a BrainNet Europe gene expression microarray study. J Neural Transm (Vienna) 122:1055-1068. https://doi.org/10.1007/ s00702-014-1293-0

34. Lesnick TG, Papapetropoulos S, Mash DC, Ffrench-Mullen J, Shehadeh L, de Andrade $\mathrm{M}$ et al (2007) A genomic pathway approach to a complex disease: axon guidance and Parkinson disease. PLoS Genet 3:e98. https:// doi.org/10.1371/journal.pgen.0030098

35. Zheng B, Liao Z, Locascio JJ, Lesniak KA, Roderick SS, Watt ML et al (2010) PGC-1alpha, a potential therapeutic target for early intervention in Parkinson's disease. Sci Transl Med 2:52-73. https://doi.org/10.1126/scitr anslmed.3001059

36. Giasson BI, Duda JE, Quinn SM, Zhang B, Trojanowski JQ, Lee VM (2002) Neuronal alpha-synucleinopathy with severe movement disorder in mice expressing A53T human alpha-synuclein. Neuron 34:521-533. https://doi. org/10.1016/s0896-6273(02)00682-7

37. Sacino AN, Brooks M, Thomas MA, McKinney AB, Lee S, Regenhardt RW et al (2014) Intramuscular injection of alpha-synuclein induces CNS alpha-synuclein pathology and a rapid-onset motor phenotype in transgenic mice. Proc Natl Acad Sci U S A 111:10732-10737. https://doi.org/10. 1073/pnas. 1321785111

38. Jan A, Jansonius B, Delaidelli A, Bhanshali F, An YA, Ferreira N et al (2018) Activity of translation regulator eukaryotic elongation factor-2 kinase is increased in Parkinson disease brain and its inhibition reduces alpha 
synuclein toxicity. Acta Neuropathol Commun 6:54. https://doi.org/10.1186/ s40478-018-0554-9

39. Gene Expression Omnibus https://www.ncbi.nlm.nih.gov/geo/

40. Ferreira N, Goncalves NP, Jan A, Jensen NM, van der Laan A, Mohseni S et al (2021) Trans-synaptic spreading of alpha-synuclein pathology through sensory afferents leads to sensory nerve degeneration and neuropathic pain. Acta Neuropathol Commun 9:31. https://doi.org/10.1186/ s40478-021-01131-8

41. Franklin KBJ, Paxinos G (2013) Paxinos and Franklin's The mouse brain in stereotaxic coordinates. Academic Press, an imprint of Elsevier, City

42. Livak KJ, Schmittgen TD (2001) Analysis of relative gene expression data using real-time quantitative PCR and the 2(-Delta Delta C(T)) method. Methods 25:402-408. https://doi.org/10.1006/meth.2001.1262

43. Jan A, Jansonius B, Delaidelli A, Somasekharan SP, Bhanshali F, Vandal M et al (2017) eEF2K inhibition blocks A beta 42 neurotoxicity by promoting an NRF2 antioxidant response. Acta Neuropathol 133:101-119. https://doi.org/ 10.1007/s00401-016-1634-1

44. Calkins MJ, Vargas MR, Johnson DA, Johnson JA (2010) Astrocyte-specific overexpression of Nrf2 protects striatal neurons from mitochondrial complex II inhibition. Toxicol Sci 115:557-568. https://doi.org/10.1093/toxsci/ $\mathrm{kfq072}$

45. PhosphoSite https://www.phosphosite.org/proteinAction.action?id=3828\& showAllsites=true

46. Numazawa S, Ishikawa M, Yoshida A, Tanaka S, Yoshida T (2003) Atypical protein kinase $\mathrm{C}$ mediates activation of NF-E2-related factor 2 in response to oxidative stress. Am J Physiol Cell Physiol 285:C334-342. https://doi.org/10. 1152/ajpcell.00043.2003

47. Fares MB, Jagannath S, Lashuel HA (2021) Reverse engineering Lewy bodies: how far have we come and how far can we go? Nat Rev Neurosci 22:111-131. https://doi.org/10.1038/s41583-020-00416-6

48. McCann H, Stevens CH, Cartwright H, Halliday GM (2014) alpha-Synucleinopathy phenotypes. Parkinsonism Relat Disord 20(Suppl 1):S62-67. https:// doi.org/10.1016/S1353-8020(13)70017-8

49. Anderson JP, Walker DE, Goldstein JM, de Laat R, Banducci K, Caccavello RJ et al (2006) Phosphorylation of Ser-129 is the dominant pathological modification of alpha-synuclein in familial and sporadic Lewy body disease. J Biol Chem 281:29739-29752. https://doi.org/10.1074/jbc.M600933200

50. Fujiwara H, Hasegawa M, Dohmae N, Kawashima A, Masliah E, Goldberg MS et al (2002) alpha-Synuclein is phosphorylated in synucleinopathy lesions. Nat Cell Biol 4:160-164. https://doi.org/10.1038/ncb748

51. Muntane G, Ferrer I, Martinez-Vicente M (2012) alpha-synuclein phosphorylation and truncation are normal events in the adult human brain. Neuroscience 200:106-119. https://doi.org/10.1016/j.neuroscience.2011.10. 042

52. Venderova K, Park DS (2012) Programmed cell death in Parkinson's disease. Cold Spring Harb Perspect Med. https://doi.org/10.1101/cshperspect.a0093 65

53. Lee $\mathrm{OH}$, Jain AK, Papusha V, Jaiswal AK (2007) An auto-regulatory loop between stress sensors INrf2 and Nrf2 controls their cellular abundance. J Biol Chem 282:36412-36420. https://doi.org/10.1074/jbc.M706517200

54. Sorrentino ZA, Xia Y, Funk C, Riffe CJ, Rutherford NJ, Ceballos Diaz C et al (2018) Motor neuron loss and neuroinflammation in a model of alphasynuclein-induced neurodegeneration. Neurobiol Dis 120:98-106. https:// doi.org/10.1016/j.nbd.2018.09.005

55. Beraud D, Hathaway HA, Trecki J, Chasovskikh S, Johnson DA, Johnson JA et al (2013) Microglial activation and antioxidant responses induced by the Parkinson's disease protein alpha-synuclein. J Neuroimmune Pharmacol 8:94-117. https://doi.org/10.1007/s1 1481-012-9401-0

56. Hsu L, Sagara Y, Arroyo A, Rockenstein E, Sisk A, Mallory M et al (2000) alphasynuclein promotes mitochondrial deficit and oxidative stress. Am J Pathol 157:401-410. https://doi.org/10.1016/s0002-9440(10)64553-1

57. Perni M, Galvagnion C, Maltsev A, Meisl G, Muller MB, Challa PK et al (2017) A natural product inhibits the initiation of alpha-synuclein aggregation and suppresses its toxicity. Proc Natl Acad Sci U S A 114:E1009-E1017. https:// doi.org/10.1073/pnas.1610586114

58. Mateo I, Infante J, Sanchez-Juan P, Garcia-Gorostiaga I, Rodriguez-Rodriguez E, Vazquez-Higuera JL et al (2010) Serum heme oxygenase-1 levels are increased in Parkinson's disease but not in Alzheimer's disease. Acta Neurol Scand 121:136-138. https://doi.org/10.1111/j.1600-0404.2009.01261.x

59. Lastres-Becker I, Ulusoy A, Innamorato NG, Sahin G, Rabano A, Kirik D et al (2012) alpha-Synuclein expression and Nrf2 deficiency cooperate to aggravate protein aggregation, neuronal death and inflammation in earlystage Parkinson's disease. Hum Mol Genet 21:3173-3192. https://doi.org/10. 1093/hmg/dds 143

60. Schipper HM, Liberman A, Stopa EG (1998) Neural heme oxygenase-1 expression in idiopathic Parkinson's disease. Exp Neurol 150:60-68. https:// doi.org/10.1006/exnr.1997.6752

61. Lastres-Becker I, Garcia-Yague AJ, Scannevin RH, Casarejos MJ, Kugler S, Rabano A et al (2016) Repurposing the NRF2 activator dimethyl fumarate as therapy against synucleinopathy in Parkinson's disease. Antioxid Redox Signal 25:61-77. https://doi.org/10.1089/ars.2015.6549

62. Skibinski G, Hwang V, Ando DM, Daub A, Lee AK, Ravisankar A et al (2017) Nrf2 mitigates LRRK2- and alpha-synuclein-induced neurodegeneration by modulating proteostasis. Proc Natl Acad Sci U S A 114:1165-1170. https:// doi.org/10.1073/pnas.1522872114

63. Tanji K, Maruyama A, Odagiri S, Mori F, Itoh K, Kakita A et al (2013) Keap1 is localized in neuronal and glial cytoplasmic inclusions in various neurodegenerative diseases. J Neuropathol Exp Neurol 72:18-28. https://doi.org/10. 1097/NEN.0b013e31827b5713

64. Yamazaki H, Tanji K, Wakabayashi K, Matsuura S, Itoh K (2015) Role of the Keap1/Nrf2 pathway in neurodegenerative diseases. Pathol Int 65:210-219. https://doi.org/10.1111/pin.12261

65. Cook AL, Vitale AM, Ravishankar S, Matigian N, Sutherland GT, Shan J et al (2011) NRF2 activation restores disease related metabolic deficiencies in olfactory neurosphere-derived cells from patients with sporadic Parkinson's disease. PLoS ONE 6:e21907. https://doi.org/10.1371/journal.pone.0021907

66. Dringen R, Pfeiffer B, Hamprecht B (1999) Synthesis of the antioxidant glutathione in neurons: supply by astrocytes of Cys Gly as precursor for neuronal glutathione. J Neurosci 19:562-569

67. Moretti D, Tambone S, Cerretani M, Fezzardi P, Missineo A, Sherman LT et al (2021) NRF2 activation by reversible KEAP1 binding induces the antioxidant response in primary neurons and astrocytes of a Huntington's disease mouse model. Free Radic Biol Med 162:243-254. https://doi.org/10.1016/j. freeradbiomed.2020.10.022

68. Fu MH, Wu CW, Lee YC, Hung CY, Chen IC, Wu KLH (2018) Nrf2 activation attenuates the early suppression of mitochondrial respiration due to the alpha-synuclein overexpression. Biomed J 41:169-183. https://doi.org/10. 1016/j.bj.2018.02.005

69. Hoenen C, Gustin A, Birck C, Kirchmeyer M, Beaume N, Felten P et al (2016) Alpha-synuclein proteins promote pro-inflammatory cascades in microglia: stronger effects of the A53T mutant. PLoS ONE 11:e0162717. https://doi. org/10.1371/journal.pone.0162717

70. O'Connell MA, Hayes JD (2015) The Keap1/Nrf2 pathway in health and disease: from the bench to the clinic. Biochem Soc Trans 43:687-689. https:// doi.org/10.1042/BST20150069

71. Ahuja M, Ammal Kaidery N, Yang L, Calingasan N, Smirnova N, Gaisin A et al (2016) Distinct Nrf2 signaling mechanisms of fumaric acid esters and their role in neuroprotection against 1-methyl-4-phenyl-1,2,3,6-tetrahydropyridine-induced experimental Parkinson's-like disease. J Neurosci 36:63326351. https://doi.org/10.1523/JNEUROSCI.0426-16.2016

72. Barone MC, Sykiotis GP, Bohmann D (2011) Genetic activation of Nrf2 signaling is sufficient to ameliorate neurodegenerative phenotypes in a Drosophila model of Parkinson's disease. Dis Model Mech 4:701-707. https://doi. org/10.1242/dmm.007575

73. Nakabeppu Y, Tsuchimoto D, Yamaguchi H, Sakumi K (2007) Oxidative damage in nucleic acids and Parkinson's disease. J Neurosci Res 85:919-934. https://doi.org/10.1002/jnr.21191

74. Delaidelli A, Jan A, Herms J, Sorensen PH (2019) Translational control in brain pathologies: biological significance and therapeutic opportunities. Acta Neuropathol 137:535-555. https://doi.org/10.1007/s00401-019-01971-8

75. Heise C, Taha E, Murru L, Ponzoni L, Cattaneo A, Guarnieri FC et al (2016) eEF2K/eEF2 pathway controls the excitation/inhibition balance and susceptibility to epileptic seizures. Cereb Cortex: https://doi.org/10.1093/cercor/ bhw075

76. Liu R, Proud CG (2016) Eukaryotic elongation factor 2 kinase as a drug target in cancer, and in cardiovascular and neurodegenerative diseases. Acta Pharmacol Sin 37:285-294. https://doi.org/10.1038/aps.2015.123

\section{Publisher's Note}

Springer Nature remains neutral with regard to jurisdictional claims in published maps and institutional affiliations. 